modifications or adaptations are made.

\title{
The Role of Environmental Distractions in the Experience of Fibrofog in Real-World Settings
}

\author{
Anna L. Kratz, ${ }^{1}$ DD Daniel Whibley, ${ }^{2}$ Samsuk Kim, ${ }^{3}$ David A. Williams, ${ }^{1}$ Daniel J. Clauw, ${ }^{1}$ and Martin Sliwinski ${ }^{4}$
}

Objective. Perceived cognitive dysfunction in people with fibromyalgia (FM), "fibrofog," is commonly reported and has been demonstrated in neurocognitive testing. Distractibility and inattention have been implicated as potential contributors to fibrofog, but the role of environmental distractions has not been explored. In this study, ambulatory assessment methods were used to examine whether FM is related to more environmental distractions and to examine the impact of distractions on subjective and objective cognitive functioning.

Methods. Fifty people with FM and 50 age-, sex-, and education-matched controls without FM completed 8 consecutive days of ambulatory assessments. Five times per day, participants reported perceived cognitive functioning and environmental distractions and completed validated tests of processing speed and working memory.

Results. The FM group reported distractions in a higher proportion of the ambulatory cognitive testing sessions (40.5\%) compared with the group without FM $(29.8 \% ; P<0.001)$ and more often reported multiple simultaneous distractions. For both groups, sound was the most common distraction. The group with FM reported more distractions caused by light, and the group without FM reported more social distractions. Group differences in subjective and objective cognitive functioning were not augmented during distraction relative to during periods of no distraction. There were no group differences in within-person changes in cognitive functioning as a function of distraction.

Conclusion. The group with FM reported more distractions than the group without FM; both groups reported poorer processing speed when distracted, and the effects of distraction on test performance did not differ significantly by group. Findings suggest that sensitivity to environmental distractions may play a role in the experience of cognitive dysfunction in FM.

\section{INTRODUCTION}

Cognitive dysfunction is reported by approximately $70 \%$ of people with fibromyalgia (FM) (1-4) and is characterized by memory problems, inattention, learning difficulties, slow processing speed, and problems with executive functioning (3-10). These cognitive problems, collectively referred to as "fibrofog," exert negative effects on perceptions about illness severity and overall mental health (5) and the ability to maintain a social network or employment and to perform a wide range of activities of daily

The contents of this article are solely the responsibility of the authors and do not necessarily represent the official views of the National Institutes of Health.

Supported by the National Institute of Arthritis and Musculoskeletal and Skin Diseases of the NIH (award K01-AR-064275; Principal Investigator Dr. Kratz). The Michigan Institute for Clinical \& Health Research (NIH award UL1-TR-002240) provided subject recruitment support through the UMHealthResearch.org website.

${ }^{1}$ Anna L. Kratz, PhD, David A. Williams, PhD, Daniel J. Clauw, MD: University of Michigan, Ann Arbor; ${ }^{2}$ Daniel Whibley, PhD: University of Michigan, Ann Arbor, and School of Medicine, Medical Sciences, and Nutrition, University of Aberdeen, Aberdeen, United Kingdom; ${ }^{3}$ Samsuk Kim, MS: University of Detroit Mercy, Detroit, Michigan ${ }^{4}$ Martin Sliwinski, PhD: Pennsylvania State University, University Park, Pennsylvania. life $(6,11)$. Fibrofog is rated among the top five most troubling FM symptoms by clinicians and patients alike (12-14). Despite its high prevalence and serious impact, cognitive dysfunction in FM is not well understood; efforts to improve or compensate for cognitive dysfunction in FM rely on new insights into what factors and processes contribute to fibrofog.

Distractibility and inattention have been strongly implicated as critical mechanisms underpinning fibrofog $(9,10)$. Chronic pain, which is characteristic of FM, may consume attentional resources, and as such, may increase susceptibility to distraction (9-10,15-19).

Dr. Kratz has received honoraria from the American Psychological Association (less than $\$ 10,000$ ). Dr. Williams has received consultant fees, speaking fees, and/or honoraria from Community Health Focus, Inc. and Swing Therapeutics, Inc. (less than $\$ 10,000$ each). Dr. Clauw has received consultant fees, speaking fees, and/or honoraria from Aptinyx, Daiichi Sankyo, Intec Pharma, Eli Lilly, Samumed, Theravance, Williams \& Connolly LLP, and Zynerba (less than $\$ 10,000$ each); he has also received consultant fees, speaking fees, and/or honoraria from Pfizer Inc., Tonix, and Nix Patterson, LLP (more than $\$ 10,000$ each). No other disclosures relevant to this article were reported.

Address correspondence to Anna L. Kratz, PhD, Department of Physical Medicine and Rehabilitation, University of Michigan, 2800 Plymouth Road, North Campus Research Complex, Building 16, G031 N, Ann Arbor, MI 48109. E-mail: alkratz@med.umich.edu.

Submitted for publication February 10, 2020; accepted in revised form February 14, 2020. 


\section{SIGNIFICANCE \& INNOVATIONS}

- To our knowledge, this is the first study to explore the role of environmental distractions in the experience of fibrofog and cognitive performance in people with fibromyalgia (FM) compared with people without FM.

- People with FM had similar declines in perceived and objective cognitive dysfunction when distracted compared with those without FM.

- People with FM reported greater environmental distractions more frequently, despite being in a familiar (eg, home) environment more of the time, compared with those without FM.

- The role of environmental distraction in the experience of fibrofog warrants further examination.

Additionally, people with FM demonstrate increased sensitivity to a variety of environmental stimuli, consistent with a hypersensitivity to sensory stimuli $(20,21)$, including temperature (22), sound (2325), visual stimuli (26), and olfactory stimuli $(27,28)$.

A single laboratory-based study examined the role of distraction on memory performance in people with or without FM (29). This study showed that when tested with no distraction during the encoding phase of a memory task, working memory ability in those with FM was largely intact; however, even a limited distraction interfered with memory recall ability in people with FM; compared with the control group, the group with FM lost 44\% more information (29). Although this single study has been held as evidence that distraction may be a critical factor underlying fibrofog, the distraction paradigm of the study does not mimic real-world distractions. First, the distraction was constrained to the "encoding" period of the memory task, with no distraction during the stimulus/learning phase of the task; distractions in the real-world do not typically pause to allow one to attend to a task. Furthermore, the distraction in the study was a single cognitive task, whereas real-world experience may include sensory stimuli, interruptions from other people, etc, or a collection of multiple simultaneous distractions.

We have previously demonstrated in a sample of adults with FM and age-, sex-, and education-matched adults without FM (non-FM) that people with FM demonstrate deficits in perceived cognitive function and objective working memory on ambulatory tests (30). As a follow-up to this primary article and to address limitations in our understanding of the role of environmental distractions in fibrofog in daily life, we will examine these ambulatory data to explore the role of environmental distractions in these FM-related deficits. We tested the following hypotheses: 1) the FM group will report environmental distractions more frequently during cognitive tests compared with the non-FM group; 2) relative to the non-FM group, the FM group will report a higher proportion of distractions caused by light, sound, and temperature; 3) group-level differences in aggregate/average subjective and objective cognitive functioning will be greater for cognitive testing sessions in which distractions were endorsed; and 4) the FM group will show steeper momentary declines (compared with the non-FM group) in cognitive functioning when they report distraction relative to when they report no distraction.

\section{PATIENTS AND METHODS}

Participants. Participants were eligible if they were 1) 18 years of age or older, 2) able to read at a sixth-grade level, and 3) able to fluently converse in English. Exclusion criteria were the following: 1) comorbid neurological disorder (eg, stroke, dementia, Parkinson disease, brain tumor, or moderate or severe traumatic brain injury), learning disorder, or cognitive impairment; 2) current substance (eg, alcohol, recreational drug) dependence or prolonged (5 years or longer) history of substance dependence; 3) visual or hearing impairment that would prevent standardized cognitive testing; 4) diagnosis of untreated obstructive sleep apnea; and 5) atypical sleep/wake patterns (eg, night-shift work schedule). Participants in the FM group were required to meet the diagnostic criteria for FM according to the 2016 American College of Rheumatology (ACR) (31), whereas participants in the non-FM group were required not to meet the diagnostic criteria for FM according to the 2016 ACR criteria. Each participant in the FM group was matched with a participant in the non-FM group based on sex, age, and education.

Study procedures. After the Medical Institutional Review Board at the University of Michigan approved all study procedures, participants were recruited through the University of Michigan from the surrounding community. Recruitment sources included existing participant registries, community groups, placement of fliers in health centers and community settings, and advertisements of the study on a university-based recruitment website (www.UMHea Ithresearch.org). Data were collected between January 2018 and August 2018.

Participation in this study involved a 90-minute baseline visit, followed by an 8-day home-monitoring period. After initial screening over the telephone for study eligibility, participants were scheduled for an in-person visit, during which they underwent informed consent procedures. During the baseline visit, participants also completed a battery of online self-report measures, completed in-person cognitive testing, and received instruction on how to use study-issued materials (wrist-worn accelerometer and smartphone). At the completion of data collection, participants returned the study devices to the laboratory via a postage-paid return box for data download and processing. Participants were compensated up to $\$ 175$ for full completion of the study. 
Data collection technology. Participants were provided a ZTE Axon 7 mini smartphone, which has a 5.2" display (1080 × 1920 pixels) and no SIM card. This device was programmed with a customized study-specific app to administer ecological momentary assessment (EMA) measures of subjective experiences and ambulatory cognitive tests. The smartphone was set up such that participants would initiate the first data collection session after waking. For the following four sessions, the app was programmed to play an audible alert to prompt the respondent to complete EMA and cognitive assessments. Alerts were programmed on a quasi-random schedule based on each participant's typical waking time, with scheduled intervals between prompts ranging between 3 and 4.5 hours. Response data were stored onboard the smartphone. Data were downloaded and processed when the participant returned it to the laboratory.

Measures. Baseline self-report measures. Participants completed a self-report demographic questionnaire assessing age, years of education, sex, and race/ethnicity, and a set of validated self-report surveys. Participants completed the Patient-Reported Outcome Measurement Information System (PROMIS) Pain Intensity 3a Short Form; participants rated worst and average pain in the past 7 days (on a 1 [no pain] to 5 [very severe] scale) and current level of pain (on a 1 [no pain] to 5 [very severe] scale), and item scores were summed and converted to a T-score metric with a mean of 50 and SD of 10 . Higher scores on the short form indicate more severe pain. The Patient Health Questionnaire-8 (PHQ-8) (32) was used to assess the frequency of eight depressive symptoms in the past 2 weeks. Scores on the $\mathrm{PHQ}-8$ range from 0 to 24 , with higher scores indicating worse depressed mood. Fatigue was assessed with a four-item short form from the PROMIS fatigue item bank that reflected the experience of fatigue (33); scores are on a T-score metric with a mean of 50 and SD of 10 . Higher scores indicate higher fatigue.

EMAs. Perceived cognitive functioning was assessed using two items from the PROMIS Applied General Concerns (34) item bank. The items, adapted for momentary assessment, include the following: "How slow is your thinking right now?" (rated on a visual analog scale [VAS] of 0-100, in which 0 indicates "my thinking is very fast" and 100 indicates "my thinking is very slow") and "How foggy is your thinking right now?" (rated on a VAS of $0-100$, in which 0 indicates "my thinking is very clear" and 100 indicates "my thinking is very foggy"). The scores were averaged to produce an aggregate score, with higher scores indicating worse perceived cognitive functioning. The internal consistency for this scale in this study was Cronbach's $a=0.95$, indicating an excellent level of reliability.

On a dichotomous scale of "yes/no", environmental distractions were assessed with the question, "During the brain games, were you distracted by anything in your environment?" If the participant selected "yes," the following checklist was provided with the instructions "please check all applicable distractions": 1) distracting sounds, 2) distracting lights, 3) distracting temperature, 4) social distractions, and 5) other distraction.

Location during testing was assessed with the question, "Where are you?" Participants were provided with the following options to choose from: 1) inside my home, 2) inside at work, 3) outdoors, and 4) other.

Ambulatory cognitive tests. In addition to the EMAs mentioned above, two brief, valid, and reliable cognitive tests (35) were administered five times a day via a study-specific smartphone app. For both ambulatory cognitive tests, response times were recorded in milliseconds.

The symbol search task is a test of processing speed. During this task, participants saw a row of four symbol pairs at the top of the screen and two symbol pairs at the bottom of the screen and decided, as quickly as possible, which symbol pair at the bottom matched one of the symbol pairs at the top and selected the matching pair by touching their selection at the bottom. Reaction time and errors were recorded. Stimuli were presented until a response was provided. Sixteen trials were administered for each testing session. Three variables were calculated for trials: average (mean) reaction time per trial, median, and the SD (variability) in reaction time per trial.

The dot memory task is a test of working memory that consists of three phases: encoding, distraction, and retrieval. During the encoding phase, the participant was asked to remember the location of three red dots appearing on a $5 \times 5$ square grid. After a 3-second examination period, the grid disappeared, and the distraction phase began. During the distraction phase, the participant was asked to locate and touch all the F's in an array of E's. After the distraction task, the retrieval phase began by presenting an empty $5 \times 5$ square grid, and the participant was asked to place the red dots (by touching the empty squares) in the correct locations. Participants were asked to press "Done" when finished. Euclidean distance, a score of the collective distance of the three dots from their correct locations, was calculated. Four trials were administered for each testing session. Average Euclidean distance, maximum Euclidean distance, and the SD of Euclidean distances for each session were calculated for this task.

Data analysis. The sample was described according to age (mean, SD), proportion of female participants, number of years of education (mean, SD), and race/ethnicity. The first day of collection of ambulatory data was excluded from all analyses, allowing participants a practice day to familiarize themselves with the cognitive tests. The percentage of eligible cognitive tests that participants completed from day 2 onward was calculated, as were percentages of testing sessions during which participants endorsed being distracted. The percentage of total tests during which each group reported being distracted was compared using a two-sample test of proportions. The sum of the number of distractions endorsed 
per testing session was calculated for each participant (eg, light and sound equaled two distractions), and distributions of these sums were plotted by group and compared using a $\chi^{2}$ test. Because of the small number of observations for which participants reported four or five distractions during a testing session, these categories were combined. The physical locations of participants during testing sessions in which they reported being distracted were summarized (number and proportion, by group) and compared using a $\chi^{2}$ test. The number and proportion of distractions by qualitative type was also summarized, with percentages per group compared using two-sample tests of proportions.

Linear regression was used to investigate group-level differences in subjective and objective cognitive functioning as a function of distraction status, with models predicting the aggregate mean value of the outcome by distraction status, including a group-by-distraction status interaction term. Unadjusted analyses were conducted in the first instance, followed by models adjusted for baseline pain (PROMIS pain intensity T-score), fatigue (PROMIS fatigue experience T-score), and depression severity (PHQ-8). Seven cognitive outcome variables were assessed in separate analyses: self-reported cognitive function, three symbol search task variables (response time mean, median, and SD), and three dot memory task variables (mean error, maximum error, and SD of Euclidean distance errors). To determine whether momentary associations between cognitive function and distraction status were moderated by group membership, multilevel level models were used (cognitive reports and contemporaneous distraction status nested within participants) that included a group-by-distraction status interaction term. Models were adjusted for age, sex, and years of education. For the six objective outcome variables, adjustments were also made for the number of times the participant had completed the cognitive task to correct for possible improvements in performance caused by practice effects. Analyses were performed using Stata (version 15; StataCorp), with a $P$ value of less than 0.05 used as the threshold to determine statistical significance.

\section{RESULTS}

Descriptive statistics. One hundred participants (50 with FM and 50 without FM) were eligible, provided informed consent, and completed baseline and repeated-measures assessments. The sample was mostly female and white, with an average age of 45 years (Table 1). The groups differed significantly in terms of mean PROMIS pain intensity T-scores $\left(F_{98}=14.52, P<0.001 ;\right.$ mean $_{\mathrm{FM}}=54.33, \mathrm{SD}=6.12$, range $=44-72 ;$ mean $_{\text {non-FM }}=35.55, \mathrm{SD}=6.81$, range $=31-58$ ). Participants were compliant with the data collection procedures, completing an average of $90.9 \%$ of all possible cognitive testing sessions; the FM group had, on average, $91.2 \%$ complete data, and the non-FM group had $90.5 \%$ complete data. Although participants were instructed to complete the ambulatory assessments for 8 consecutive days, some participants completed a few additional assessments (eg, kept the phone through the morning of the ninth day). All available data were examined in these analyses. As a result, there were very small differences in the number of cognitive testing sessions administered to the two groups because of minor variations in the number of days/sessions for which the participants provided data.

Between-group comparison of environmental distractions during cognitive tests. Of 1654 cognitive testing sessions, the FM group provided distraction ratings for 1633 (98.7\%). Of 1629 cognitive testing sessions, the non-FM group provided distraction ratings for 1617 (99.2\%). The FM group reported being distracted during $40.5 \%$ of these sessions ( $n=661$ ), whereas the non-FM group reported being distracted for $29.8 \%$ of these sessions ( $n=481$ ), a statistically significant difference (mean difference: 10.7\% [95\% confidence interval 7.5\%14.0\%]; SE $=0.02 ; z=6.41 ; P<0.001)$.

Within the 661 distracted sessions for the FM group, a total of 1117 distraction sources were endorsed. Within the 481 distracted sessions for the non-FM group, a total of 724 distraction sources were endorsed. There was a statistically significant relationship between the sum of distractions per distracted testing

Table 1. Participant demographic characteristics

\begin{tabular}{lccc}
\hline & $\begin{array}{c}\text { Total Participants } \\
(\mathrm{N}=100)\end{array}$ & $\begin{array}{c}\text { Participants With FM } \\
(\mathrm{n}=50)\end{array}$ & $\begin{array}{c}\text { Participants Without } \\
\text { FM }(\mathrm{n}=50)\end{array}$ \\
\hline Age, y & & & \\
$\quad$ Mean (SD) & $45.1(13.9)$ & $44.9(13.9)$ & $45.2(14.0)$ \\
$\quad$ Range & $18-73$ & $20-70$ & $18-73$ \\
Female sex, $\mathrm{n}(\%)$ & $88(88)$ & $44(88)$ & $44(88)$ \\
Education, yean (SD) & $15.7(2.0)$ & $15.7(2.0)$ & $15.8(2.0)$ \\
Race, $\mathrm{n}(\%)$ & & & $38(76)$ \\
White & $81(81)$ & $43(86)$ & $8(16)$ \\
Black & $13(13)$ & $5(10)$ & $1(2)$ \\
Biracial or multiracial & $3(3)$ & $2(4)$ & $3(6)$ \\
Asian & $3(3)$ & $0(0)$ & \\
\hline
\end{tabular}

Abbreviation: FM, fibromyalgia. 


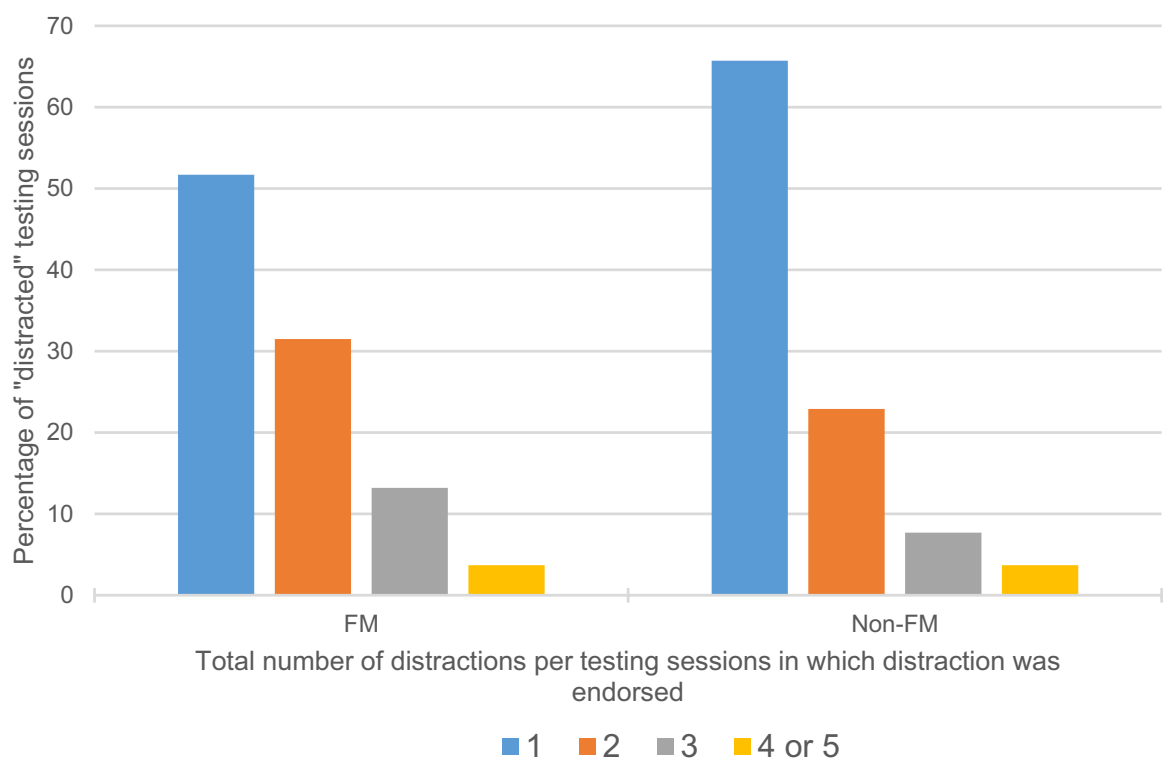

Figure 1. Histograms of the percentage of testing sessions in which distractions were endorsed with 1, 2, 3, or 4 to 5 total concurrent distractions for the group with fibromyalgia $(F M)(n=661$ distracted sessions) and the group without fibromyalgia $(n o n-F M)(n=481$ distracted sessions).

session and group membership, with the FM group reporting a significantly higher number of sources of distraction per session $\left(X^{2}=24.7 ; P<0.001\right)$ (Figure 1). For example, across the distracted sessions, the FM group reported two distractions $31.5 \%$ of the time and three distractions $13.2 \%$ of the time. In contrast, the non-FM group reported two distractions $22.9 \%$ of the time and three distractions $7.7 \%$ of the time during distracted sessions.

Physical location during distracted tests. The FM group was more frequently at home and less frequently outdoors during distracted sessions compared with the non-FM group (Table 2). Results of a $\chi^{2}$ test indicated a statistically significant relationship between location when distracted and group membership $\left(\chi^{2}=10.00 ; P=0.02\right)$.

Proportion of distraction type by group. The most common source of distraction reported during cognitive tests by both groups was sound. The FM group reported statistically significant higher proportions of distractions due to light and other distractions, whereas the non-FM group was more likely to endorse social distractions (Table 3).

Table 2. Frequency of distracted sessions by location and group

\begin{tabular}{lccc}
\hline \multicolumn{1}{c}{ Location } & FM & Non-FM & Total \\
\hline At home & $422(63.8 \%)$ & $283(58.8 \%)$ & 705 \\
At work & $84(12.7 \%)$ & $56(11.6 \%)$ & 140 \\
Outdoors & $43(6.5 \%)$ & $56(11.6 \%)$ & 99 \\
Other & $112(16.9 \%)$ & $86(17.9 \%)$ & 198 \\
Total & 661 & 481 & 1142 \\
\hline
\end{tabular}

Abbreviation: FM, fibromyalgia.
Group-level differences in subjective and objective cognitive functioning as a function of distraction. There were no significant interactions between group membership and distraction status for subjective or objective measures of cognitive function (all $P>0.70$ ). This finding was consistent after adjusting for baseline levels of pain intensity, fatigue, and depression (all $P>0.66)$.

Group differences in momentary changes in subjective and objective cognitive functioning as a function of distraction. Multilevel model results indicated a significant group-by-distraction status interaction for subjective cognitive functioning ( $B=-2.22$; $\mathrm{SE}=0.99 ; z=-2.24 ; P=0.025$ ), whereas momentary distraction status was not associated with concurrent self-reported cognitive function for the FM group (contrast test -0.01 ; SE $=0.72 ; z=-0.01 ; P=0.99$ ); for the non-FM group, being distracted was associated with slightly better selfreported cognitive function (contrast test -2.23 ; $\mathrm{SE}=0.66$; $z=-3.37 ; P=0.001)$. Although statistically significant, the effect was modest. There were no significant interactions between group membership and distraction status for any momentary measures of objective cognitive function (all $P<0.34$ ).

There were main effects of distraction on objective cognitive function. In multilevel models predicting cognitive test performance from distraction status, controlling for group and pre-stated covariates, there were significant main effects for poorer mean reaction time SD ( $B=108.80 ; \mathrm{SE}=26.76 ; z=4.07 ; P<0.001)$, mean error $(B=0.30 ; \mathrm{SE}=0.05 ; z=6.53 ; P<0.001)$, maximum error $(B=0.43$; SE $=0.08 ; z=5.58 ; P<0.001)$, and error SD $(B=0.11 ; \mathrm{SE}=0.04 ; z=3.00 ; P=0.003)$ during distracted 
Table 3. No. and percentage of distractions by type

\begin{tabular}{lccc}
\hline \multicolumn{1}{c}{ Distraction Source } & FM, n (\%) & Non-FM, n (\%) & $\begin{array}{c}\text { Two-Sample Test } \\
\text { of Proportions, } P\end{array}$ \\
\hline Light & $70(6.3)$ & $27(3.7)$ & 0.003 \\
Temperature & $63(5.6)$ & $39(5.4)$ & 0.41 \\
Social & $270(24.2)$ & $236(32.6)$ & 0.006 \\
Sound & $431(38.6)$ & $295(40.7)$ & 0.18 \\
Other & $283(25.3)$ & $127(17.5)$ & $<0.001$ \\
Total & $1117(100)$ & $724(100)$ & $\ldots$ \\
\hline
\end{tabular}

Abbreviation: FM, fibromyalgia.

sessions relative to nondistracted session. There were no main effects of distraction on tests of working memory.

\section{DISCUSSION}

One prevailing hypothesis about mechanisms underlying fibrofog is that increased sensitivity to external distractions, such as light and sound, is an important contributor to difficulty attending to cognitive tasks for people with $\operatorname{FM}(9,21)$. This is the first study to examine the experience of environmental distractions during cognitive work in the lived-in environment, comparing people with and without FM. People with FM reported more distractions more frequently compared with those without FM; this includes more frequent reporting of multiple simultaneous distractions. This set of findings is consistent with the expectation that people with FM will perceive more distractions in the lived-in environment because of perceptual amplification and generalized hypersensitivity to both internal and external stimuli (21).

This increase in the frequency of perceived distractions was paired with an expected decrease in cognitive performancespecifically, processing speed-in the context of distractions. The expectation that those with FM would demonstrate a more robust decline in cognitive performance and in perceived cognitive functioning in the context of distractions compared with those without FM was not supported. Both groups demonstrated decline in cognitive performance on ambulatory tests during distractions and were not different in the scope of this decline. This is in conflict with evidence from existing literature showing that individuals with FM tend to have particular difficulty minimizing the co-occurrence or consequences of distractions from stimulus competition, which has led to decreased working memory and processing information and impaired executive functioning $(7-8,10,29)$. Brain imaging studies suggest that the decreased task-related brain activity in people with FM represents a deficit in the inhibition network and increased activation in other brain regions, which suggests competing resources resulting in reduced resources for staying attentive and performing tasks (19). The reduced attentional resources can lead to greater susceptibility to distraction and slow information processing (10). This initial conflicting finding that people with FM are not especially susceptible to cognitive effects of distraction warrants replication and extension in studies that are more sophisticated in terms of how distractions and cognition are assessed in real-world settings. Future studies that pair ambulatory assessment of cognition with neuroimaging techniques could provide new insights into how brain function/connectivity relate to day-to-day distractibility and fibrofog.

Interestingly, and in contrast with expectations, ratings of perceived cognitive dysfunction did not differ based on distraction status for those with FM and were lower during distracted sessions for those without FM. The EMA items of perceived cognitive dysfunction assessed mental clarity and speed; it is possible that when people without FM reported low levels of cognitive dysfunction, they were signaling a sense of alertness or awareness that made them more perceptive of environmental distractions.

These findings reveal a number of interesting contextual details about distractions and cognition in FM. The finding that people with FM report more environmental distractions is particularly striking given that they were more likely than those without FM to report being distracted while at home and less likely to be distracted while outdoors. This makes sense in the context of findings that chronic pain, including FM-related pain, contributes to disability, isolation, and social withdrawal $(36,37)$. This study did not track participants' locations, so it is not possible to know why individuals with FM were more likely to report distractions in a familiar environment (home) and less likely to report distractions outdoors; this finding warrants further examination. Although sound was the most common source of distraction for both groups, it is notable that people with FM were more likely to report distractions from light; again, this is interesting in the context of these distractions occurring in the home rather than outdoors, as was more common in the non-FM group.

The focus of this article was on the role of distractions perceived in the external environment. People with FM may also be subject to more internal distractions or interference from sensory and emotional experiences, such as pain, fatigue, and depressed mood, and the influence of these internal experiences on fibrofog is the focus of a separate analysis of these data. However, a broader range of potentially impactful factors, such as FM-related alterations in brain structure and function (eg, gray matter volume and neuroinflammation) needs to be examined to fully understand the underlying mechanisms of cognitive dysfunction in FM and to identify potentially fruitful preventive, 
restorative, and compensatory strategies to address fibrofog. Comparisons of common and distinct mechanisms of cognitive dysfunction across different conditions associated with cognitive difficulties (eg, cancer treated with chemotherapy, clinical depression) are also likely to increase our understanding of poor cognitive functioning in people with FM.

This article provides an important first look at the role of environmental distractions in fibrofog in the daily lives of people with FM; however, the findings must be interpreted in the context of a number of limitations. Effort on the cognitive tests was not assessed using traditional tests of motivation; however, as noted in the primary article, high accuracy on the processing speed test and lack of global deficits on ambulatory tests (30) does not support the notion that the people with FM were "faking bad" or demonstrating poor effort relative to the controls without FM. The study relied solely on self-report of distractions, which does not capture objective levels of environmental stimulation. Future studies would ideally incorporate continuous passive measurement of ambient temperature, noise, light, etc to objectively assess the environment. Future studies could also incorporate experimentally administered distractions, such as lights and sounds from the testing device, for some of the test sessions. Collection of objective data on environmental distractions as well as administration of experimentally induced distractions would also provide the opportunity to examine the psychometrics of the ambulatory self-reported distractions measures, which is sorely needed. Another limitation is that the two cognitive tests assessed a very limited range of cognitive functioning domains; higher-level cognitive domains, such as aspects of executive functioning and cognitive flexibility, might be more sensitive to the effects of distraction than the tests of processing speed and working memory, which are relatively lower-level cognitive domains. Future studies in larger samples that include other non-FM populations with chronic pain (such as those with arthritis or headache) or people with sensory sensitivity but without chronic pain would provide additional insights into the mechanisms underlying brain fog including fibrofog.

Despite similar declines in objective cognitive functioning when distracted, relative to those without FM, evidence from this study suggests that people with FM do experience more distractions more often in their environment. Findings support the idea that sensitivity to environmental stimulation may play a role in the cognitive problems in everyday life for those with FM.

\section{AUTHOR CONTRIBUTIONS}

All authors drafted the article, revised it critically for important intellectual content, approved the final version to be published, and take responsibility for the integrity of the data and the accuracy of the data analysis.

Study conception and design. Kratz, Sliwinski.

Acquisition of data. Kratz, Sliwinski.

Analysis and interpretation of data. Kratz, Whibley, Kim, Williams, Clauw.

\section{REFERENCES}

1. Park DC, Glass JM, Minear M, Crofford LJ. Cognitive function in fibromyalgia patients. Arthritis Rheum 2001;44:2125-33.

2. Kravitz HM, Katz RS. Fibrofog and fibromyalgia: a narrative review and implications for clinical practice. Rheumatol Int 2015;35:1115-25.

3. Tesio V, Torta DM, Colonna F, Leombruni P, Ghiggia A, Fusaro E, et al. Are fibromyalgia patients cognitively impaired? Objective and subjective neuropsychological evidence. Arthritis Care Res (Hoboken) 2015;67:143-50.

4. Bertolucci $\mathrm{PH}$, de Oliveira FF. Cognitive impairment in fibromyalgia. Curr Pain Headache Rep 2013;17:344.

5. Katz RS, Heard AR, Mills M, Leavitt F. The prevalence and clinical impact of reported cognitive difficulties (fibrofog) in patients with rheumatic disease with and without fibromyalgia. J Clin Rheumatol 2004;10:53-8.

6. Arnold LM, Crofford LJ, Mease PJ, Burgess SM, Palmer SC, Abetz $\mathrm{L}$, et al. Patient perspectives on the impact of fibromyalgia. Patient Educ Couns 2008;73:114-20.

7. Miró E, Lupiáñez J, Hita E, Martínez M, Sánchez A, Buela-Casal G. Attentional deficits in fibromyalgia and its relationships with pain, emotional distress and sleep dysfunction complaints. Psychol Health 2011;26:765-80.

8. Wu YL, Huang CJ, Fang SC, Ko LH, Tsai PS. Cognitive impairment in fibromyalgia: a meta-analysis of case-control studies. Psychosom Med 2018;80:432-8.

9. Bell T, Trost Z, Buelow MT, Clay O, Younger J, Moore D, et al. Metaanalysis of cognitive performance in fibromyalgia. $J$ Clin Exp Neuropsychol 2018;40:698-714.

10. Teodoro T, Edwards MJ, Isaacs JD. A unifying theory for cognitive abnormalities in functional neurological disorders, fibromyalgia and chronic fatigue syndrome: systematic review. J Neurol Neurosurg Psychiatry 2018;89:1308-19.

11. Dennis NL, Larkin M, Derbyshire SW. 'A giant mess': making sense of complexity in the accounts of people with fibromyalgia. $\mathrm{Br} \mathrm{J}$ Health Psychol 2013;18:763-81.

12. Bennett RM, Jones J, Turk DC, Russell IJ, Matallana L. An internet survey of 2,596 people with fibromyalgia. BMC Musculoskelet Disord 2007;8:27.

13. Mease PJ, Arnold LM, Crofford LJ, Williams DA, Russell IJ, Humphrey $L$, et al. Identifying the clinical domains of fibromyalgia: contributions from clinician and patient Delphi exercises. Arthritis Rheum 2008;59:952-60.

14. Mease P. Fibromyalgia syndrome: review of clinical presentation, pathogenesis, outcome measures, and treatment. J Rheumatol Suppl 2005;75:6-21.

15. Seminowicz DA, Davis KD. A re-examination of pain-cognition interactions: implications for neuroimaging. Pain 2007;130:8-13.

16. Seminowicz DA, Davis KD. Pain enhances functional connectivity of a brain network evoked by performance of a cognitive task. J Neurophysiol 2007;97:3651-9.

17. Glass JM, Williams DA, Fernandez-Sanchez ML, Kairys A, Barjola $\mathrm{P}$, Heitzeg MM, et al. Executive function in chronic pain patients and healthy controls: different cortical activation during response inhibition in fibromyalgia. J Pain 2011;12:1219-29.

18. Leavitt F, Katz RS, Mills M, Heard AR. Cognitive and dissociative manifestations in fibromyalgia. J Clin Rheumatol 2002;8:77-84.

19. Dick B, Eccleston C, Crombez G. Attentional functioning in fibromyalgia, rheumatoid arthritis, and musculoskeletal pain patients. Arthritis Rheum 2002;47:639-44.

20. Lorenz J, Grasedyck K, Bromm B. Middle and long latency somatosensory evoked potentials after painful laser stimulation in patients with fibromyalgia syndrome. Electroencephalogr Clin Neurophysiol 1996;100:165-8. 
21. McDermid AJ, Rollman GB, McCain GA. Generalized hypervigilance in fibromyalgia: evidence of perceptual amplification. Pain 1996;66:133-44.

22. Kosek E, Ekholm J, Hansson P. Sensory dysfunction in fibromyalgia patients with implications for pathogenic mechanisms. Pain 1996;68:375-83.

23. Carrillo-de-la-Pena MT, Vallet M, Perez M, Gomez-Perretta C. Intensity dependence of auditory-evoked cortical potentials in fibromyalgia patients: a test of the generalized hypervigilance hypothesis. $J$ Pain 2006;7:480-7.

24. Hollins M, Harper D, Gallagher S, Owings EW, Lim PF, Miller V, et al. Perceived intensity and unpleasantness of cutaneous and auditory stimuli: an evaluation of the generalized hypervigilance hypothesis. Pain 2009;141:215-21.

25. Petzke F, Clauw DJ, Ambrose K, Khine A, Gracely RH. Increased pain sensitivity in fibromyalgia: effects of stimulus type and mode of presentation. Pain 2003;105:403-13.

26. Harte SE, Ichesco E, Hampson JP, Peltier SJ, Schmidt-Wilcke T, Clauw DJ, et al. Pharmacologic attenuation of cross-modal sensory augmentation within the chronic pain insula. Pain 2016;157:1933-45.

27. Lötsch J, Kraetsch HG, Wendler J, Hummel T. Self-ratings of higher olfactory acuity contrast with reduced olfactory test results of fibromyalgia patients. Int J Psychophysiol 2012;86:182-6.

28. Lombion S, Comte A, Tatu L, Brand G, Moulin T, Millot JL. Patterns of cerebral activation during olfactory and trigeminal stimulations. Hum Brain Mapp 2009;30:821-8.

29. Leavitt F, Katz RS. Distraction as a key determinant of impaired memory in patients with fibromyalgia. J Rheumatol 2006;33:127-32.
30. Kratz AL, Whibley D, Kim S, Sliwinski M, Clauw DJ, Williams DA. Fibrofog in daily life: an examination of ambulatory subjective and objective cognitive function in fibromyalgia. Arthritis Care Res (Hoboken) 2019. E-pub ahead of print.

31. Wolfe F, Clauw DJ, Fitzcharles MA, Goldenberg DL, Hauser W, Katz $\mathrm{RL}$, et al. 2016 revisions to the 2010/2011 fibromyalgia diagnostic criteria. Semin Arthritis Rheum 2016;46:319-29.

32. Kroenke K, Strine TW, Spitzer RL, Williams JB, Berry JT, Mokdad $\mathrm{AH}$. The PHQ-8 as a measure of current depression in the general population. J Affect Disord 2009;114:163-73.

33. Kratz AL, Schilling S, Goesling J, Williams DA. The PROMIS FatigueFM Profile: a self-report measure of fatigue for use in fibromyalgia Qual Life Res 2016;25:1803-13.

34. Cella D, Yount S, Rothrock N, Gershon R, Cook K, Reeve B, et al. The Patient-Reported Outcomes Measurement Information System (PROMIS): progress of an $\mathrm{NIH}$ Roadmap cooperative group during its first two years. Med Care 2007;45 Suppl 1: S3-11.

35. Sliwinski MJ, Mogle JA, Hyun J, Munoz E, Smyth JM, Lipton RB. Reliability and validity of ambulatory cognitive assessments. Assessment 2018;25:14-30.

36. Bolwijn PH, van Santen-Hoeufft MH, Baars HM, van der Linden S. Social network characteristics in fibromyalgia or rheumatoid arthritis. Arthritis Care Res 1994;7:46-9.

37. Wallace LS, Wexler RK, McDougle L, Miser WF, Haddox JD. Voices that may not otherwise be heard: a qualitative exploration into the perspectives of primary care patients living with chronic pain. J Pain Res 2014;7:291-9. 